\title{
Human decidua mesenchymal stem cells regulate decidual natural killer cell function via interactions between collagen and leukocyte-associated immunoglobulin-like receptor 1
}

\author{
QIANG FU ${ }^{1 *}$, XUEJING MAN ${ }^{2 *}$, MIN YU $^{3}$, YONGLI CHU $^{2}$, XIYING LUAN ${ }^{1}$, HAILAN PIAO ${ }^{3}$, \\ JIANGNAN XUE $^{1}$ and CHANGZHU JIN ${ }^{1}$ \\ ${ }^{1}$ Department of Immunology, College of Basic Medicine, Binzhou Medical University, Yantai, Shandong 264003; \\ ${ }^{2}$ Department of Clinical Medicine, Yantai Yuhuangding Hospital, Yantai, Shandong 264000; ${ }^{3}$ Laboratory of \\ Reproductive Immunology, Obstetrics and Gynecology Hospital, Fudan University, Shanghai 200011, P.R. China
}

Received April 5, 2016; Accepted May 15, 2017

DOI: $10.3892 / \mathrm{mmr} .2017 .6921$

\begin{abstract}
The development of maternal tolerance to the fetal allograft in critical for the maintenance of the pregnancy, and it is accompanied by the development of a special decidual natural killer (dNK) cell tolerance phenotype. To understand the factors that influence dNK cells during early pregnancy, the present study aimed to identify mesenchymal stem cells (MSCs) from human first-trimester deciduas, termed decidual MSCs (DMSCs), and to investigate the effect of DMSCs on the regulation of dNK cells via collagen. Decidual samples were collected from women with normal pregnancy that had undergone elective vaginal surgical terminations at 6-9 weeks gestation. DMSCs derived from human decidual tissues were cultured under differentiation conditions to examine their multipotent differentiation capacities, and the expression of MSC-specific markers, including cluster of differentiation (CD)44, CD73, CD105, CD90, CD34, CD31, CD14, CD45, $\mathrm{CD} 11 \mathrm{~b}$ and human leukocyte antigen-antigen $\mathrm{D}$ related, was determined. dNK cells were co-cultured with DMSCs in order to examine the effect of DMSCs on the tolerance phenotype of dNK cells. The expression of cell surface molecules, natural cytotoxicity triggering receptor 3 and killer cell immunoglobulin-like receptor (KIR) 2DL1, and the secretion of cytokines, including interferon- $\gamma$, tumor necrosis factor (TNF)- $\alpha$, interleukin (IL)-10, IL-4 and perforin, were examined by flow cytometry analysis. To determine whether the regulation of dNK cells by DMSCs was mediated by collagen, DMSCs were
\end{abstract}

Correspondence to: Professor Changzhu Jin, Department of Immunology, College of Basic Medicine, Binzhou Medical University, 346 Guanhai Road, Yantai, Shandong 264003, P.R. China E-mail: jincz63@hotmail.com

\section{${ }^{*}$ Contributed equally}

Key words: mesenchymal stem cells, natural killer cells, collagen, leukocyte-associated immunoglobulin-like receptor 1 pre-treated with human recombinant leukocyte-associated immunoglobulin-like receptor (LAIR)-2 and transfected with pScoR-GFP-hP4H to inhibit the interaction between LAIR-1 and collagen. The present results demonstrated that collagen produced by DMSCs increased the expression of KIR2DL1 and IL-4, decrease the expression of NKp30 and TNF- $\alpha$. In conclusion, the results of the present study demonstrated that DMSCs may be cultured in vitro for prolonged periods, whilst retaining the ability to differentiate into different cell lineages. In addition, DMSCs may modulate the function of dNK cells via the interaction between collagen and LAIR-1.

\section{Introduction}

Previous studies have demonstrated that mesenchymal stem cells (MSCs) may be isolated from muscle, adipose and endometrial tissues, as well as umbilical cord blood, and are able to be induced to undergo osteogenic and adipogenic differentiation (1-4). The primary function of MSCs is considered to be the maintenance of local tissue remodeling (1-4). Following observations that MSCs are weakly immunogenic and exhibit immunosuppressive effects on the adaptive and innate immune systems, MSCs have become a particular interest to immunologists (5).

The invasion of trophoblasts induces the differentiation of endometrial stromal cells to form the decidua, which is a highly specialized structure consisting of stromal cells, glandular cells and leukocytes $(6,7)$. The primary function of the human decidua is to ensure optimal conditions for implantation of the embryo and placentation (8). In addition, the decidua is thought to function as an active immune regulation partner for the immune tolerance microenviroment at the maternal-fetal interface. It has been proposed that a population of stem cells exists within the human decidua, which may be responsible for mediating cell proliferation during embryonic implantation and the formation of the placenta (9). The maintenance of pregnancy involves maternal tolerance to the fetal allograft, which is associated with decidual natural killer cell (dNK) tolerance phenotype (10) and a T-helper (Th) 2 cell bias at the maternal-fetal interface (11). Spaggiari and Moretta (12) 
reviewed what is known regarding the interactions between human MSCs and NK cells. It was demonstrated that the function and proliferation of NK cells is inhibited by MSCs (12). However, decidual MSCs (DMSCs) are not well characterized, and, to the best of the author's knowledge, the effect of DMSCs on the phenotype and function of dNK cells has not been previously investigated. In the present study, DMSCs were isolated from early human decidua and were first confirmed to be MSCs by examining the expression of specific cell surface markers. These cells were then employed to investigate the ability of DMSCs to regulate the phenotype and biological function of dNK cells.

\section{Materials and methods}

Human decidual tissue collection. All procedures involving study participants were approved by the Human Research Ethics Committee of Binzhou Medical University (Yantai, China), and written informed consent was received from all subjects for the collection and use of their tissue samples for the purposes of the study. Human decidual tissues were obtained from 3 women that had undergone elective vaginal termination of first-trimester pregnancies (gestational age, 6-9 weeks; age, 24-26 years) for non-medical reasons between October and November 2014 in Yuhuangding Hospital (Yantai, China). All tissues were immediately collected and stored in ice-cold Dulbecco's modified Eagle's medium (DMEM; Invitrogen; Thermo Fisher Scientific, Inc., Waltham, MA, USA), transported to the laboratory within $60 \mathrm{~min}$ following surgery and washed in DMEM containing $100 \mathrm{U} / \mathrm{ml}$ penicillin and $100 \mathrm{mg} / \mathrm{ml}$ streptomycin (Beijing Solarbio Science \& Technology Co., Ltd., Beijing, China).

Isolation and culture of DMSCs and $d N K$ cells. Decidual tissues were minced into $>1 \mathrm{~mm}^{3}$ pieces. Following an additional wash with PBS (pH 7.4), the pieces were cultured in complete DMEM/F12 medium supplemented with $10 \%$ fetal bovine serum (FBS) (both from Gibco; Thermo Fisher Scientific, Inc.), $100 \mathrm{U} / \mathrm{ml}$ penicillin and $100 \mu \mathrm{g} / \mathrm{ml}$ streptomycin in $5 \%$ $\mathrm{CO}_{2}$ at $37^{\circ} \mathrm{C}$. Following 5 days of culture, large sections were removed and adherent cells were maintained in culture with medium replenishment every third day. Cells were visualized under a microscope and identified using MSC-specific markers with flow cytometry as described below, and cells at passages 3-10 were employed for downstream experiments.

Decidual tissues were minced into $1 \mathrm{~mm}^{3}$ pieces and enzymatically digested for $20 \mathrm{~min}$ at $37^{\circ} \mathrm{C}$, under vigorous agitation, with $1.5 \mathrm{mg}$ DNase type I and $24 \mathrm{mg}$ collagenase type IV (Sigma-Aldrich; Merck KGaA; Darmstadt, Germany) in 15 ml RPMI-1640 medium (Gibco; Thermo Fisher Scientific, Inc.). Following digestion, decidual immune cells (DICs) were isolated and purified by discontinuous Percoll gradient centrifugation, using the methods described previously (13). The concentration of DICs obtained ranged between 1.062 and $1.077 \mathrm{~g} / \mathrm{ml}$, and were subsequently collected and cultured in complete RPMI-1640 medium supplemented with 10\% FBS, $100 \mathrm{U} / \mathrm{ml}$ penicillin and $100 \mu \mathrm{g} / \mathrm{ml}$ streptomycin in $5 \% \mathrm{CO}_{2}$ at $37^{\circ} \mathrm{C}$. Following primary culture for $30 \mathrm{~min}$ at $37^{\circ} \mathrm{C}$ in $5 \% \mathrm{CO}_{2}$, the adherent decidual stromal cells were removed, leaving DICs that were $98 \%$ pure. A total of $4 \times 10^{7} \mathrm{dNK}$ cells were purified with microbeads conjugated to the anti-human CD56 monoclonal antibody, using the CD56 MultiSort kit (cat no. 130-055-401; Miltenyi Biotec GmbH, Bergisch Gladbach, Germany). Separation was performed using the autoMACS ${ }^{\circledR}$ Pro separator with Volumes software (version 2.0.1.5; Miltenyi Biotec $\mathrm{GmbH}$ ), as previously described (14).

Osteogenic and adipogenic differentiation of DMSCs. The osteogenic differentiation of DMSCs was performed using OriCell $^{\mathrm{TM}}$ Mesenchymal Stem Cell Osteogenic Differentiation Medium (cat no. GUXMX-90021; Cyagen Biosciences, Inc., Santa Clara, CA, USA). DMSCs at passage 4 were detached following treatment with a solution containing $0.05 \%$ trypsin and $1 \mathrm{mM}$ EDTA, washed in PBS and then seeded at a final density of $1 \times 10^{4}$ cells $/ \mathrm{cm}^{2}$ in 24 -well plates in triplicate. At $80-90 \%$ confluency, the growth medium was carefully aspirated from each well, and $1 \mathrm{ml}$ osteogenic differentiation medium was added. Cells were cultured at $37^{\circ} \mathrm{C}$ and $5 \% \mathrm{CO}_{2}$, and the medium was refreshed every 3 days. The presence of calcium-containing osteocytes was determined following 21 days of exposure to osteogenic differentiation medium by staining the cells with alizarin red $\mathrm{S}$. To achieve this, the osteogenic differentiation medium was first removed and cells were rinsed with PBS and fixed with $4 \%$ formaldehyde solution at $20^{\circ} \mathrm{C}$ for $30 \mathrm{~min}$. The cells were subsequently stained with a working solution of $1 \%$ alizarin red $\mathrm{S}$ at $20^{\circ} \mathrm{C}$ for 3-5 min, followed by washing with distilled water to remove any unbound dye.

The adipogenic differentiation of DMSCs was performed using OriCell ${ }^{\mathrm{TM}}$ Mesenchymal Stem Cell Adipogenic Differentiation Medium (cat no. GUXMX-90031; Cyagen Biosciences, Inc.). DMSCs at passage 4 were seeded at a concentration of $1 \times 10^{4}$ cells $/ \mathrm{cm}^{2}$ in 24-well plates in triplicate. When the cells were $100 \%$ confluent, the growth medium was aspirated from the wells, and $0.5 \mathrm{ml}$ OriCell ${ }^{\mathrm{TM}}$ Mesenchymal Stem Cell Adipogenic Differentiation Medium was added. Following 3 days in culture, the medium was replaced with adipogenic differentiation medium $\mathrm{B}$, and cells were incubated for a further $24 \mathrm{~h}$. Following 4 cycles of induction and maintenance, the cells were cultured in adipogenic differentiation medium B for an additional 7 days, and subsequently stained with oil red $\mathrm{O}$. To achieve this, the adipogenic differentiation medium was first removed, the cells were rinsed with PBS and then fixed with $4 \%$ formaldehyde solution at $20^{\circ} \mathrm{C}$ for $30 \mathrm{~min}$. The cells were subsequently stained with a $0.6 \%$ oil red $\mathrm{O}$ solution at $20^{\circ} \mathrm{C}$ for $30 \mathrm{~min}$, followed by washing with distilled water to remove the unbound dye. Control cells from the same passage were cultured in DMEM supplemented with 10\% FBS and stained with oil red $\mathrm{O}$ according to the same protocol.

Prolyl-4-hydroxylase (P4H) short hairpin RNA (shRNA) plasmid transfection. Collagen molecules are trimeric polypeptide chains that form a triple helix. Hydroxylation of proline residues present in the polypeptides is catalyzed by collagen $\mathrm{P} 4 \mathrm{H}$ and is essential for triple helix formation and stability (15). In the present study, 5x10 4 DMSCs were seeded in 24-well plates with DMEM supplemented with $10 \%$ FBS. When cells had reached $85 \%$ confluence, $2 \mu 1$ Lipofectamine ${ }^{\circledR} 2000$ (Invitrogen; Thermo Fisher Scientific, Inc.), $50 \mu 1$ Opti-MEM ${ }^{\mathrm{TM}}$ (Gibco; Thermo Fisher Scientific, Inc.) 
and 20 pmol pScoR-GFP-hP4H shRNA plasmid (GeneChem Co., Ltd., Shanghai, China) were mixed and incubated at $20^{\circ} \mathrm{C}$ for $20 \mathrm{~min}$, and subsequently added to the cells according to the manufacturer's protocol (Lipofectamine ${ }^{\circledR} 2000$; Invitrogen; Thermo Fisher Scientific, Inc.). The vector-only pScoR-GFP plasmid (Shanghai GeneChem Co., Ltd.) was used as a negative control. Non-transfected DMSCs were treated as the blank controls. Following $6 \mathrm{~h}$ of incubation at $37^{\circ} \mathrm{C}$, the plasmid vector-containing medium was replaced with DMEM/F12 supplemented with $10 \%$ FBS and cells were cultured in 5\% $\mathrm{CO}_{2}$ at $37^{\circ} \mathrm{C}$.

Reverse transcription-quantitative polymerase chain reaction $(R T-q P C R)$. The effect of transfection on $\mathrm{P} 4 \mathrm{H}$ expression in DMSCs was determined by RT-qPCR, using the $2^{-\Delta \Delta C q}$ method for quantification of $\mathrm{P} 4 \mathrm{H}$ expression (16). Total RNA was extracted from DMSCs using TRIzol ${ }^{\circledR}$ reagent (Invitrogen; Thermo Fisher Scientific, Inc.), according to the manufacturer's protocol. Total RNA $(1 \mu \mathrm{g})$ was denatured, and reverse transcribed into cDNA for $1 \mathrm{~h}$ at $42^{\circ} \mathrm{C}$ with $0.5 \mu \mathrm{g}$ oligo(dT) 18, $1.0 \mathrm{mM}$ 4dNTP, $20 \mathrm{U}$ RNasin RNase inhibitor (Promega Corporation, Madison, WI, USA), 200 U Moloney virus-reverse transcriptase (SuperScript II; Thermo Fisher Scientific, Inc.), and $5 \mathrm{X}$ reaction buffer, in a reaction volume of $20 \mu \mathrm{l}$. Amplification was performed with SYBR-Green PCR Master Mix (PerkinElmer, Inc., Waltham, MA, USA), $0.8 \mathrm{mM}$ specific sense and antisense primers, and $5 \mu \mathrm{l} \mathrm{cDNA}$ in a $50 \mu \mathrm{l}$ reaction volume in a $\mathrm{T} 100^{\mathrm{TM}}$ thermal cycler (Bio-Rad Laboratories, Inc., Hercules, CA, USA ). The primers used for the detection of P4H were as follows: Forward, 5'-CTGCGG GACCTGACTAGATT-3' and reverse, 5'-TGCTCCACCTTC TCATAGCC-3'; and for $\beta$-actin were: Forward, 5'-CCCTGG ACTTCGAGCAAGAG-3' and reverse, 5'-TCTCCTTCTGCA TCCTGTCG-3'. After a 5-min pre-cycle at $95^{\circ} \mathrm{C}$, the reaction was followed by 30 cycles at $94^{\circ} \mathrm{C}$ for $1 \mathrm{~min}$, at $55^{\circ} \mathrm{C}$ for $1 \mathrm{~min}$, and at $72^{\circ} \mathrm{C}$ for $1 \mathrm{~min}$. A final extension step at $72^{\circ} \mathrm{C}$ for $15 \mathrm{~min}$ was performed. Relative gene expression was normalized to $\beta$-actin. All experiments were repeated three times.

Cell co-culture unit. Control DMSCs and those transfected with empty vector or pScoR-GFP-hP4H shRNA were seeded in 24-well plates at a density of $1 \times 10^{5}$ cells/well. In this co-culture unit, dNK cells were subsequently directly added to the wells at the same density following $12 \mathrm{~h}$ of co-culture. DMSCs and dNK cells ( $1 \times 10^{5}$ cells/well) were cultured alone as controls. A total volume of $1 \mathrm{ml}$ DMEM/F-12 supplemented with $10 \%$ FBS was added to each well. A total of $6 \mathrm{~h}$ prior to the addition of $\mathrm{dNK}$ cells, $10 \mu \mathrm{g} / \mathrm{ml}$ leukocyte-associated immunoglobulin-like receptor 2 (LAIR-2; cat no. ab182705; Abcam, Cambridge, UK) was added to specific co-culture wells. Floating dNK cells were collected for flow cytometry analysis following $48 \mathrm{~h}$ of co-culture.

Flow cytometry. Monoclonal antibodies targeting the following proteins: CD44 (cat no. 555478), CD73 (cat no. 561254), CD105 (cat no. 561443), CD34 (cat no. 555821), CD31/platelet endothelial cell adhesion molecule (PECAM-1; cat no. 555445), CD14 (cat no. 555397), CD45 (cat no. 555482), CD305/LAIR-1 (cat no. 550,811), CD56 (cat no. 555516) and CD3 (cat no. 561809) were obtained from BD Biosciences
(Franklin Lakes, NJ, USA); monoclonal antibodies targeting CD90 (cat no. A15761), CD11b (cat no. 11-0113-42), and human leukocyte antigen-antigen D related (HLA-DR; cat no. 11-9952-42) were purchased from Thermo Fisher Scientific, Inc. Multi-color flow cytometric analysis was performed using a FACSCalibur flow cytometer (BD Biosciences). dNK cells were resuspended in PBS at a density of $1 \times 10^{7} / \mathrm{ml}$, then aliquoted (100 $\mu \mathrm{l} /$ tube) to Falcon round bottom polystyrene tubes (BD Biosciences). The expression of surface molecules on NK cells was detected by triplicate labeling with fluorescein isothiocyanate (FITC)-conjugated anti-human CD56, phycoerythrin (PE)-conjugated anti-human CD3 and PE-cyanine 5.5-conjugated anti-human LAIR-1, Alexa Fluor 647-conjugated anti-natural cytotoxicity triggering receptor 3 (NKp30; cat no. 558408; BD Biosciences) and allophycocyanin (APC)-conjugated anti-killer cell immunoglobulin-like receptor 2DL1 (KIR2DL1; cat no. 564319; BD Biosciences) or their corresponding isotype controls: FITC mouse immunoglobulin (Ig) G1, $\kappa$ isotype control (cat no. 556649) and PE mouse IgG1, $\kappa$ isotype control (cat no. 551436) (both from BD Biosciences). For intracellular molecule detection, $\mathrm{CD} 3{ }^{-\mathrm{CD}} 56^{+} \mathrm{NK}$ cells were gated and the expression of perforin, interferon (IFN) $-\gamma$, tumor necrosis factor (TNF)- $\alpha$, interleukin (IL)-4 and IL-10 was determined using APC-conjugated anti-perforin (cat no. 563576), anti-IFN- $\gamma$ (cat no. 563495) anti-TNF- $\alpha$ (cat no. 551384), anti-IL-4 (cat no. 561233) or anti-IL-10 (cat no. 564372) antibodies (BD Biosciences). Cells were fixed and permeabilized at $4^{\circ} \mathrm{C}$ for $20 \mathrm{~min}$ with Fixation/Permeabilization Solution kit (cat no. 554715; BD Cytofix/Cytoperm ${ }^{\mathrm{TM}}$; BD Biosciences) according to the manufacturer's protocol. All antibodies were incubated with DMSCs or dNK cells at $4^{\circ} \mathrm{C}$ for $30 \mathrm{~min}$ with the recommended volumes at a 1:10 dilution. The levels of each molecule were subsequently analyzed using a FACSCalibur Flow Cytometer (BD Biosciences) and BD CellQuest software version 5.1 (BD Biosciences). Post-acquisition fluorescence-activated cell sorting results were analyzed using FlowJo software version 9.9.5 (FlowJo LLC, Inc., Ashland, OR, USA).

Collagen assay. DMSCs were plated in a 24-well plate at a density of $1 \times 10^{5}$ cells/well overnight in DMEM supplemented with $10 \%$ FBS; P4H shRNA or the control plasmid was added to the medium according to the protocols listed above. Following $48 \mathrm{~h}$, the cells were centrifuged at 1,000 x $\mathrm{g}$ for $10 \mathrm{~min}$ at $20^{\circ} \mathrm{C}$. Collagen IV levels in sample supernatants were quantified using an enzyme-linked immunosorbent assay analysis using commercial kit (cat no. SEA180Hu; Wuhan USCN Business Co., Ltd., Wuhan, China) according to the manufacturer's instructions. The absorbance of each well was measured using a DigiScan microplate reader (Tecan Group Ltd., Männedorf, Switzerland) at a wavelength of $450 \mathrm{~nm}$.

Total collagen produced by DMSCs was analyzed using an amino acid analyzer (Biochrom, Ltd., Cambridge, UK). Briefly, DMSCs were centrifuged at $1,000 \mathrm{x}$ g for $10 \mathrm{~min}$ at $20^{\circ} \mathrm{C}$ and the supernatant was hydrolyzed in $6 \mathrm{M} \mathrm{HCl}$ at $110^{\circ} \mathrm{C}$ for $22 \mathrm{~h}$. The samples were subsequently cooled, dried, filtered and analyzed on a Biochrom 30+ Amino Acid Analyzer according to the manufacturer's instructions (Biochrom, Ltd.). Hydroxyproline was identified against an amino acid 

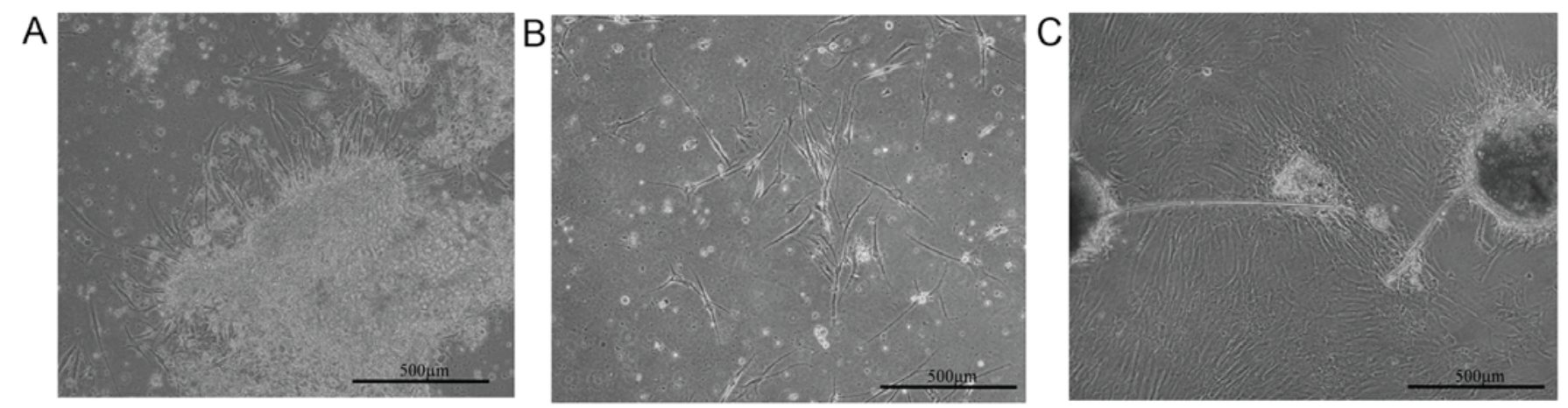

Figure 1. Light microscopy analysis of the morphology of fibroblast-like cells from human deciduas. (A) Presence of MSC-like cells emerging from the decidua tissue sections following 5 days of culture. (B) Clear presence of MSC-like cells at 5 days following the removal of the decidua tissue sections. (C) MSC-like cells proliferated into a morphologically homogenous layer of cells that adhere to the plastic surface at $\sim 10$ days of culture. Scale bar, $500 \mu \mathrm{m}$. MSC, mesenchymal stem cell.
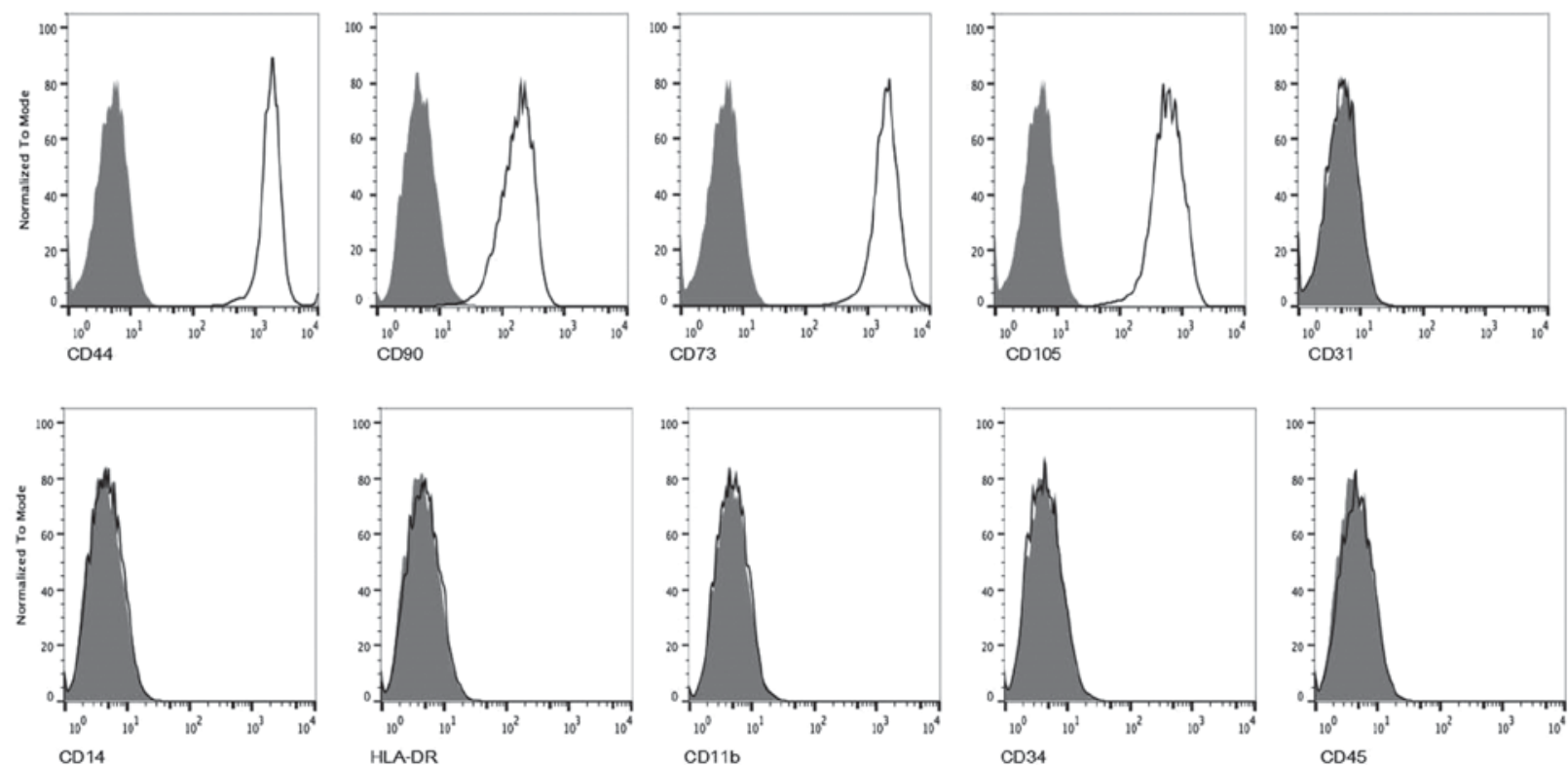

Figure 2. Analysis of cell surface markers of DMSCs by flow cytometry. Histograms showing the expression of cell surface markers demonstrated that DMSCs were positive for CD44, CD90, CD73 and CD105. This indicated that DMSCs were not contaminated by hematopoietic cell lineages, which possess CD14, HLA-DR, CD11b, CD34, CD31 and CD45 cell surface markers. DMSCs, decidual mesenchymal stem cells.

standard, and the total quantity of collagen in each sample was calculated.

Statistical analysis. Statistical analysis was performed by one-way or two-way analysis of variance. The post hoc Dunnett's test was used to compare significance levels between the control and various treatment groups. Statistical analysis was performed using SPSS software version 13.0 (SPSS, Inc., Chicago, IL, USA). The results are presented as the mean + standard error of three replicates. $\mathrm{P}<0.05$ was considered to indicate a statistically significant difference.

\section{Results}

Microscopically-defined morphology of DMSCs. Light microscopy was employed to examine the morphology of fibroblast-like cells from deciduas following 5 days of culture and prior to the removal of decidua pieces (Fig. 1A). Following
5 days of culture, the decidua tissue sections were removed and MSC-like cells were observed (Fig. 1B). These cells exhibited a characteristic spindle-shape and were adherent to the plastic culture vessel (Fig. 1B). The MSC-like cells proliferated to form a morphologically homogenous and adherent layer of cells following 10 days (Fig 1C). At this point the cells were referred to as DMSCs (Fig. 1C).

Identification of DMSCs by immunophenotypes and multilineage capacity. DMSCs at passage 5 were analyzed by flow cytometry for the expression of specific cell lineage markers. DMSCs were negative for hematopoietic and endothelial antigens CD45, CD34, CD14, CD31, CD11b and HLA-DR, while they were positive for the MSCs markers CD44, CD90, CD73 and CD105 (Fig. 2) (1,2,5). It has been previously demonstrated that MSCs may be induced to differentiate along the adipogenic and osteogenic lineages using specific culture media (17). In the present study, DMSCs were cultured in 
A

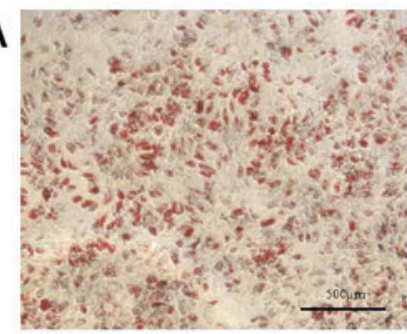

B

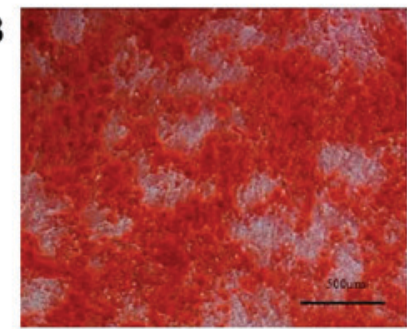

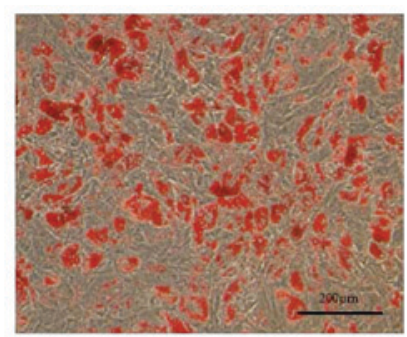
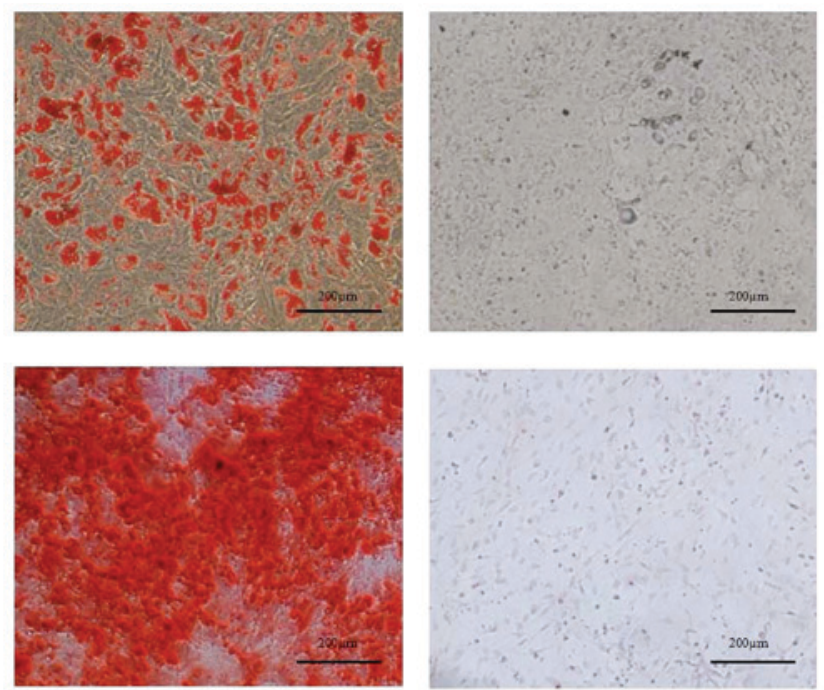

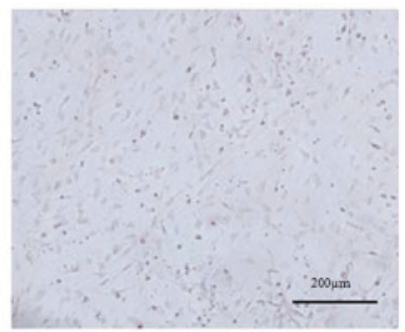

Figure 3. Multilineage differentiation capacity of DMSCs. DMSCs were cultured in (A) osteogenic differentiation medium for 21 days prior to alizarin red S staining or (B) adipogenic differentiation media for 23 days prior to oil red O staining. Cells in the left panel were stained positively, with scale bars, $500 \mu \mathrm{m}$; those in the middle panel were stained positively, with scale bars, $200 \mu \mathrm{m}$; and those in the right panel were stained negatively as a control, with scale bars, $200 \mu \mathrm{m}$. DMSCs, decidual mesenchymal stem cells.

B

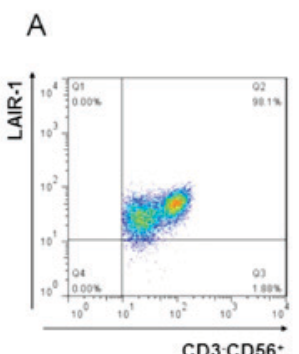

C

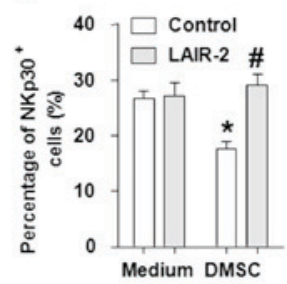

F

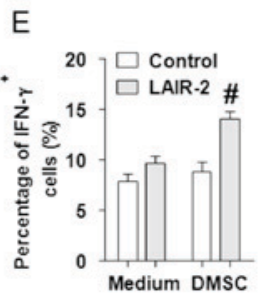

D

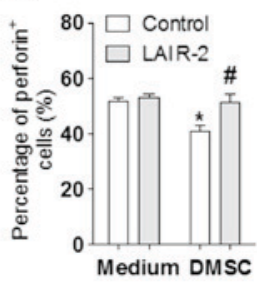

G

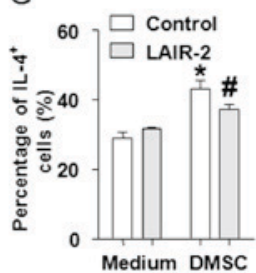

Figure 4. DMSCs modulate the phenotype and cytokine production of dNK cells via collagen secretion. (A) Purified dNK cells were identified by flow cytometry analysis. dNKs were cultured alone or with DMSCs, and in the presence or absence of recombinant human LAIR-2. Following culture for 48 h, cells were harvested and labeled with fluorescence-conjugated antibodies. Flow cytometry was performed to detect the expression of (B) KIR2DL1 and (C) NKp30 cell surface molecules, as well as (D) perforin, (E) IFN- $\gamma$, (F) TNF- $\alpha$ and (G) IL-4 intracellular molecules in dNK cells. The results are presented as the mean + standard error of the mean $(n=3)$ using different decidua samples. ${ }^{*} \mathrm{P}<0.05$ vs. control dNKs cultured in medium only; ${ }^{\#} \mathrm{P}<0.05$ vs. control dNKs co-cultured with non-pretreated DMSCs. DMSCs, decidual mesenchymal stem cells; dNK, decidual natural killer cells; LAIR-2, leukocyte-associated immunoglobulin-like receptor 2; KIR2DL1, killer cell immunoglobulin-like receptor 2DL1; NKp30, natural cytotoxicity triggering receptor 3; IFN, interferon; TNF, tumor necrosis factor; IL, interleukin.

adipogenic or osteogenic differentiation medium, and stained with oil red $\mathrm{O}$ (Fig. 3A) and alizarin red S (Fig. 3B) to confirm successful differentiation into adipocytes and osteocytes, respectively. Therefore, the isolated DMSCs met the essential criteria used to define MSCs.

DMSCs regulate dNK cells via collagen secretion. The present study investigated whether dNK cells may be regulated by DMSCs. The expression of CD56 and LAIR-1 on dNK cells purified with microbeads was first analyzed by flow cytometry. The results demonstrated that the percentage of CD56- and
LAIR-1-positive cells was $>98 \%$ (Fig. 4A). dNK cells were then co-cultured with DMSCs for 2 days, before the floating cells were harvested for analysis of NK cell phenotype and the expression of intracellular cytokines by flow cytometry. The expression of NKp30 and perforin was significantly decreased, while KIR2DL1 expression was significantly increased in dNK cells co-cultured with DMSCs when compared with dNK cells alone (Fig. 4B-D). In addition, the intracellular cytokine expression profile of TNF- $\alpha$ and IL- 4 was significantly altered following co-culture of dNK cells with DMSCs, whereas IFN- $\gamma$ levels were not (Fig. 4E-G). As expected, the observed 
A

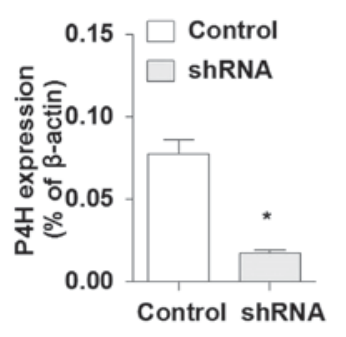

B

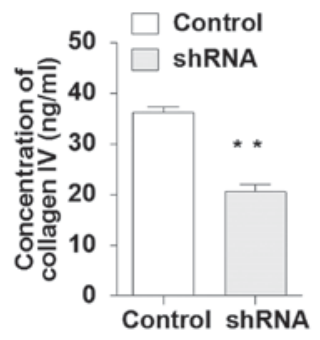

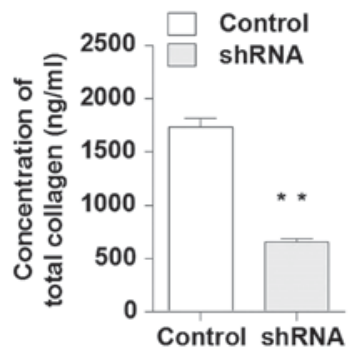

Figure 5. Knockdown of collagen expression in DMSCs. DMSCs were seeded in a 24-well plate and incubated overnight prior to transfection with P4H shRNA or an empty vector control plasmid. At $48 \mathrm{~h}$ following transfection, the supernatant and cells were harvested separately. (A) Reverse transcription-quantitative polymerase chain reaction analysis was employed to examine the mRNA levels of P4H. (B) Enzyme-linked immunosorbent assay analysis was performed to analyze the protein levels of collagen IV. (C) An amino acid analyzer was used to detect total collagen levels. The results are presented as the mean + standard error of the mean $(\mathrm{n}=3)$ using different decidua samples. ${ }^{*} \mathrm{P}<0.05$ and ${ }^{* *} \mathrm{P}<0.01$ vs. control. DMSCs, decidual mesenchymal stem cells; $\mathrm{P} 4 \mathrm{H}$, prolyl-4-hydroxylase; shRNA, short hairpin RNA.
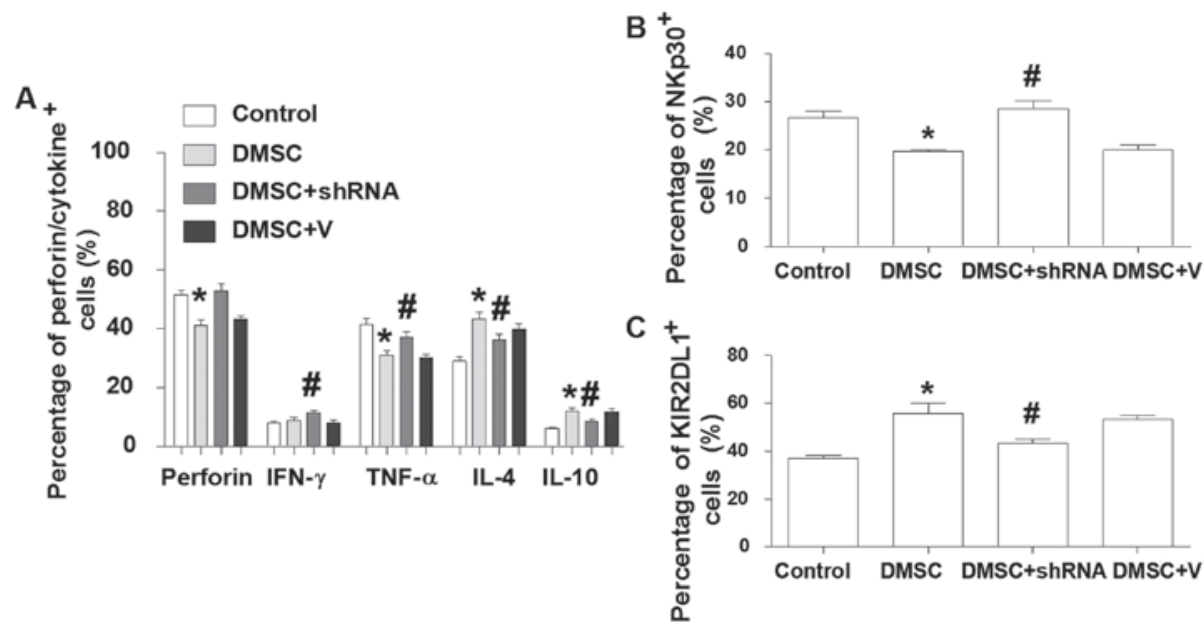

Figure 6. Inhibition of collagen expression in DMSCs abrogates DMSC-induced alterations in the phenotype and cytokine production of dNK cells. Purified dNK cells were cultured alone, with DMSCs or with DMSCs transfected with prolyl-4-hydroxylase shRNA or empty vector controls. Following 48-h of culture, floating dNK cells were harvested and labeled with fluorescence-conjugated antibodies. Flow cytometry analysis was employed to detect the expression of the (A) intracellular molecules IFN- $\gamma$, TNF- $\alpha$ and IL-4, and the cell surface molecules (B) KIR2DL1 and (C) NKp30 in dNK cells. The results are presented as the mean + standard error of the mean $(n=3)$ using different decidua samples. " $\mathrm{P}<0.05$ vs. control and ${ }^{\#} \mathrm{P}<0.05$ vs. DMSC $+\mathrm{V}$. DMSCs, decidual mesenchymal stem cells; dNK, decidual natural killer cells; shRNA, short hairpin RNA; KIR2DL1, killer cell immunoglobulin-like receptor 2DL1; NKp30, natural cytotoxicity triggering receptor 3; IFN, interferon; TNF, tumor necrosis factor; IL, interleukin; V, empty vector plasmid.

effects of DMSCs on dNK cells were abrogated by LAIR-2 pretreatment, indicating that DMSCs may regulate dNK cells via the interaction between collagen and LAIR-1.

Knockdown of collagen expression in DMSCs affects the phenotype and cytokine expression profile of $d N K$ cells. To confirm whether collagen produced by DMSCs was responsible for the observed effects of DMSCs on dNK cells, the expression of collagen in DMSCs was silenced by transfection with an shRNA specific to the $\beta$-subunit of P4H. RT-qPCR analysis demonstrated that $\mathrm{P} 4 \mathrm{H}$ mRNA levels were reduced by $>70 \%$ in P4H-shRNA-transfected cells when compared with empty vector controls (Fig. 5A). In addition, secretion of total collagen and collagen IV was significantly reduced in P4H-shRNA-transfected cells when compared with control cells (Fig. 5B and C). Furthermore, flow cytometry analysis demonstrated that interference with collagen expression in DMSCs abrogated the effects of DMSCs on dNK cells. As shown in Fig. 6A, the expression levels of perforin, IFN- $\gamma$ and
TNF- $\alpha$ were increased in dNKs, and the expression of IL-4 and IL-10 was decreased following co-culture of dNKs with DMSCs transfected with P4H shRNA for 2 days. In addition, the expression of NKp30 was significantly increased, while KIR2DL1 was significantly reduced in dNKs co-cultured with DMSCs transfected with P4H shRNA when compared with the non-transfected co-culture group (Fig. 6B and C).

\section{Discussion}

The primary function of the decidua is to ensure that optimal conditions are provided for embryonic implantation and placentation. It has been hypothesized that MSCs originating from human bone marrow are recruited via the circulation to the endometrium, where they proliferate and differentiate under the influence of the specific environment supported by reproductive hormones and growth factors (8). It has been previously reported that MSCs may inhibit NK cell proliferation and their cytotoxic activities (18); however, the isolation 
of MSCs from the decidua and the regulation of dNK cells by DMSCs has not been previously investigated.

In the present study, DMSCs with a fibroblast-like morphology, were cultured in vitro and exhibited sustained growth for $>10$ passages. The DMSCs exhibited a phenotype that is usually ascribed to cells of mesenchymal origin, as evidenced by the positive expression of CD44, CD90, CD73 and CD105 markers and the absence of hematopoietic cell markers. The results indicated that co-culture of dNKs with DMSCs reduced the expression of NKp30 and increased the percentage of KIR2DL1-expressing dNK cells when compared with dNKs cultured alone. In addition, co-culture with DMSCs downregulated perforin, IFN- $\gamma$ and TNF- $\alpha$ production, and upregulated IL-4 and IL-10 expression by dNK cells.

It has been previously established that collagen is important for generating a microenvironment that supports trophoblast survival and migration (14). A previous study reported that, during normal pregnancy, type IV collagen is detected in decidual cells by pericellular immunostaining (19). However, upon spontaneous abortion, a weak or complete absence of type IV collagen staining was observed in the cells (19). In addition, the decidual tissues from patients that had undergone a spontaneous abortion exhibited reduced total collagen expression (14). Although dNK cells utilize collagen for migration and retention at the maternal-fetal interface (20), the immunoregulatory effects of collagen on DICs requires further investigation. It has been previously reported that collagens produced by tumor cell lines are capable of activating LAIR-1, and may induce immune tolerance in the tumor microenviroment (21). However, whether DMSCs at the maternal-fetal interface induce immune tolerance via collagen remains unknown. LAIR-1 is a member of the immunoglobulin superfamily and contains two immunoreceptor tyrosine-based inhibition motifs in its intracellular domain that are required to convey inhibitory signals to NK cells (22). LAIR-2 is a potent inhibitor of the interaction between the LAIR-1 and collagen $(23,24)$. The results of the present study demonstrated that pretreatment of $\mathrm{dNK}$ cells with LAIR-2 abrogated the effects of DMSCs on the cytotoxic phenotype and proinflammatory cytokine secretion of dNK cells.

Collagen triple helix integrity is dependent on proline hydroxylation by $\mathrm{P} 4 \mathrm{H}$. It has been reported that LAIR-1 may bind to the collagen triple helix peptides that contain multiple glycine-proline-hydroxyproline repeats (25). In the present study, the post-transcriptional modification of collagen was disrupted by transfecting DMSCs with $\mathrm{P} 4 \mathrm{H}$ shRNA, as this leads to insufficient proline hydroxylation of collagen and subsequently affects the ability of collagen to bind LAIR-1. Knockdown of P4H in DMSCs induced alterations in the phenotype and cytokine expression profile of dNKs, resulting in a proinflammatory milieu. The switch from a Th2 to Th1 cytokine profile at the maternal-fetal interface is harmful for the maintenance of successful pregnancy (13). The results of the present study demonstrated that DMSCs may serve an important role in maintaining a Th2 bias at the maternal-fetal interface, and that this may be achieved via collagen. These results are consistent with a previous report demonstrating that the collagen-specific $\mathrm{CD}^{+} \mathrm{T}$-cell response is altered from a dominant $\mathrm{Th} 0 / \mathrm{Th} 1$ response to a Th2 phenotype in vivo (26).
In the majority of maternal DICs, various immune inhibitory receptors are expressed that function to prevent excessive immune responses to paternal alloantigens originating from trophoblasts (14). However, further studies regarding the mechanisms involved in maternal-fetal immune tolerance during normal pregnancy are required. The activity of NK cells depends on the balance between inhibitory and activating receptors. It has been demonstrated that maternal KIR2DL1 serves a protective role for the fetus $(27,28)$. The results of the current study demonstrated that DMSCs may induce the expression of KIR2DL1 via collagen, and that the activity of dNKs may be affected when a dominant KIR2DL1-mediated inhibitory signal is not received due to reduced collagen expression at the maternal-fetal interface. Natural cytotoxicity receptors (NCRs) are markers that regulate NK cell cytotoxicity and cytokine production. It has been previously reported that an increase in the percentage of $\mathrm{NKp} 30^{+} \mathrm{NK}$ cells is observed in the decidual tissue of patients that exhibit embryonic implantation failure and recurrent miscarriages (29). Conversely, in patients with successful pregnancies, the expression of NKp30 is significantly reduced (30). In addition, NCRs and NK1-derived cytokines have been demonstrated to be downregulated in normal fertile women (29). The results of the current study are consistent with a previous study demonstrating that NKp30 mediates the secretion of IFN- $\gamma$ and TNF- $\alpha$ in the decidua during early pregnancy (31). When combined with the observed decrease of perforin in $\mathrm{dNKs}$ co-cultured with DMSCs, these results indicate that DMSCs may contribute to the reduced cytotoxicity of dNKs via collagen at the maternal-fetal interface.

In conclusion, the present study reports a novel method to isolate multipotent DMSCs from first-trimester human decidua tissues. The results demonstrated that DMSCs exhibit clonogenic properties, are able to be cultured in vitro for prolonged periods, differentiate into different cell lineages and express cell surface markers specific to MSCs. In addition, when co-cultured with dNKs, DMSCs exhibited LAIR-1-mediated inhibition of TNF- $\alpha$ and perforin secretion and NKp30 expression, thus suggesting a LAIR-1-mediated tolerance phenotype of dNK cells via collagen, which may contribute to the induction of an immune-tolerant microenvironment at the maternal-fetal interface.

\section{Acknowledgements}

The present study was supported by the Nature Science Foundation of China (grant nos. 81370730, 81571512, 81273200, 31370905 and 31300751), the Nature Science Foundation of Shandong Province (grant nos. ZR2015JL027 and ZR2011HQ006) and the Science and Technology Plan of Binzhou Medical University (grant no. BY2007KYQD02).

\section{References}

1. Reyes M,Lund T,Lenvik T, Aguiar D, Koodie L and Verfaillie CM: Purification and ex vivo expansion of postnatal human marrow mesodermal progenitor cells. Blood 98: 2615-2625, 2001.

2. Zvaifler NJ, Marinova-Mutafchieva L, Adams G, Edwards CJ, Moss J, Burger JA and Maini RN: Mesenchymal precursor cells in the blood of normal individuals. Arthritis Res 2: 477-488, 2000 . 
3. Zuk PA, Zhu M, Ashjian P, De Ugarte DA, Huang JI, Mizuno H, Alfonso ZC, Fraser JK, Benhaim P and Hedrick MH: Human adipose tissue is a source of multipotent stem cells. Mol Biol Cell 13: 4279-4295, 2002

4. Schwab KE and Gargett CE: Co-expression of two perivascular cell markers isolates mesenchymal stem-like cells from human endometrium. Hum Reprod 22: 2903-2911, 2007.

5. Ma S, Xie N, Li W, Yuan B, Shi Y and Wang Y: Immunobiology of mesenchymal stem cells. Cell Death Differ 21: 216-225, 2014.

6. Kammerer U, von Wolff M and Markert UR: Immunology of human endometrium. Immunobiology 209: 569-574, 2004

7. Poehlmann TG, Schaumann A, Busch S, Fitzgerald JS Aguerre-Girr M, Le Bouteiller P, Schleussner E and Markert UR: Inhibition of term decidual NK cell cytotoxicity by soluble HLA-G1. Am J Reprod Immunol 56: 275-285, 2006.

8. Dimitrov R, Kyurkchiev D, Timeva T, Yunakova M, Stamenova M, Shterev A and Kyurkchiev S: First-trimester human decidua contains a population of mesenchymal stem cells. Fertil Steril 93: 210-219, 2010

9. Guo C, Zhu H, Huang W, Li S, Qu W, Liu Y and Tan A: Side population cells in the human decidua of early pregnancy exhibit stem/progenitor cell-like characteristics. Reprod Biomed Online 21: 783-793, 2010.

10. Nakashima A, Shima T, Inada K, Ito M and Saito S: The balance of the immune system between T cells and NK cells in miscarriage. Am J Reprod Immunol 67: 304-310, 2012.

11. Zhu XY, Zhou YH, Wang MY, Jin LP, Yuan MM and Li DJ: Blockade of CD86 signaling facilitates a Th2 bias at the maternal-fetal interface and expands peripheral $\mathrm{CD} 4^{+} \mathrm{CD} 25$ regulatory $\mathrm{T}$ cells to rescue abortion-prone fetuses. Biol Reprod 72: 338-345, 2005.

12. Spaggiari GM and Moretta L: Cellular and molecular interactions of mesenchymal stem cells in innate immunity. Immunol Cell Biol 91: 27-31, 2013

13. Piao HL, Tao Y, Zhu R, Wang SC, Tang CL, Fu Q, Du MR and Li DJ: The CXCL12/CXCR4 axis is involved in the maintenance of Th2 bias at the maternal/fetal interface in early human pregnancy. Cell Mol Immunol 9: 423-430, 2012.

14. Fu Q, Tao Y, Piao H, Du MR and Li DJ: Trophoblasts and decidual stromal cells regulate decidual NK cell functions via interaction between collagen and LAIR-1. Am J Reprod Immunol 71: 368-378, 2014.

15. Qi HH, Ongusaha PP, Myllyharju J, Cheng D, Pakkanen O, Shi Y, Lee SW, Peng J and Shi Y: Prolyl 4-hydroxylation regulates Argonaute 2 stability. Nature 455: 421-424, 2008.

16. Livak KJ and Schmittgen TD: Analysis of relative gene expression data using real-time quantitative PCR and the 2(-Delta Delta C(T)) method. Methods 25: 402-408, 2001.

17. Bae YJ, Kwon YR, Kim HJ, Lee S and Kim YJ: Enhanced differentiation of mesenchymal stromal cells by three-dimensional culture and azacitidine. Blood Res 52: 18-24, 2017.

18. Pradier A, Passweg J, Villard J and Kindler V: Human bone marrow stromal cells and skin fibroblasts inhibit natural killer cell proliferation and cytotoxic activity. Cell Transplant 20: 681-691, 2011

19. Iwahashi M, Muragaki Y, Ooshima A and Nakano R: Decreased type IV collagen expression by human decidual tissues in spontaneous abortion. J Clin Endocrinol Metab 81: 2925-2929, 1996.
20. Burrows TD, King A and Loke YW: The role of integrins in adhesion of decidual NK cells to extracellular matrix and decidual stromal cells. Cell Immunol 166: 53-61, 1995.

21. Rygiel TP, Stolte EH, de Ruiter T, van de Weijer ML and Meyaard L: Tumor-expressed collagens can modulate immune cell function through the inhibitory collagen receptor LAIR-1. Mol Immunol 49: 402-406, 2011.

22. Meyaard L, Adema GJ, Chang C, Woollatt E, Sutherland GR, Lanier LL and Phillips JH: LAIR-1, a novel inhibitory receptor expressed on human mononuclear leukocytes. Immunity 7 : 283-290, 1997.

23. Lebbink RJ, van den Berg MC, de Ruiter T, Raynal N, van Roon JA, Lenting PJ, Jin B and Meyaard L: The soluble leukocyte-associated Ig-like receptor (LAIR)-2 antagonizes the collagen/LAIR-1 inhibitory immune interaction. J Immunol 180 1662-1669, 2008

24. Lenting PJ, Westerlaken GH, Denis CV, Akkerman JW and Meyaard L: Efficient inhibition of collagen-induced platelet activation and adhesion by LAIR-2, a soluble Ig-like receptor family member. PLoS One 5: e12174, 2010.

25. Lebbink RJ, Raynal N, de Ruiter T, Bihan DG, Farndale RW and Meyaard L: Identification of multiple potent binding sites for human leukocyte associated Ig-like receptor LAIR on collagens II and III. Matrix Biol 28: 202-210, 2009.

26. Doncarli A, Stasiuk LM, Fournier C and Abehsira-Amar O Conversion in vivo from an early dominant Th0/Th1 response to a Th2 phenotype during the development of collagen-induced arthritis. Eur J Immunol 27: 1451-1458, 1997.

27. Kusnierczyk P: Killer cell immunoglobulin-like receptor gene associations with autoimmune and allergic diseases, recurrent spontaneous abortion, and neoplasms. Front Immunol 4: 8, 2013.

28. Flores AC, Marcos CY, Paladino N, Arruvito L, Williams F, Middleton D and Fainboim L: KIR receptors and HLA-C in the maintenance of pregnancy. Tissue Antigens 69 (Suppl 1): S112-S113, 2007.

29. Fukui A, Ntrivalas E, Fukuhara R, Fujii S, Mizunuma H, Gilman-Sachs A, Beaman K and Kwak-Kim J: Correlation between natural cytotoxicity receptors and intracellular cytokine expression of peripheral blood NK cells in women with recurrent pregnancy losses and implantation failures. Am J Reprod Immunol 62: 371-380, 2009.

30. Marlin R, Duriez M, Berkane N, de Truchis C, Madec Y, Rey-Cuille MA, Cummings JS, Cannou C, Quillay $\mathrm{H}$, Barré-Sinoussi F, et al: Dynamic shift from CD85j/ILT-2 to NKG2D NK receptor expression pattern on human decidual NK during the first trimester of pregnancy. PLoS One 7: e30017, 2012.

31. El Costa H, Casemayou A, Aguerre-Girr M, Rabot M, Berrebi A, Parant O, Clouet-Delannoy M, Lombardelli L, Jabrane-Ferrat N, Rukavina D, et al: Critical and differential roles of NKp46-and NKp30-activating receptors expressed by uterine NK cells in early pregnancy. J Immunol 181: 3009-3017, 2008. 